Published in final edited form as:

Arch Intern Med. 2006 February 27; 166(4): 465-471.

\title{
Risk of the "Androgen Deprivation Syndrome" in Men Receiving Androgen Deprivation for Prostate Cancer
}

\author{
Vahakn B. Shahinian, MD, MS, Yong-Fang Kuo, PhD, Jean L. Freeman, PhD, and James S. \\ Goodwin, MD \\ Departments of Internal Medicine (Drs Shahinian, Kuo, Freeman, and Goodwin) and Preventive \\ Medicine and Community Health (Drs Kuo, Freeman, and Goodwin), and Sealy Center on Aging \\ (Drs Shahinian, Kuo, Freeman, and Goodwin), University of Texas Medical Branch, Galveston
}

\begin{abstract}
Background-Androgen deprivation therapy for prostate cancer has been associated with a spectrum of adverse effects, such as depression, memory difficulties, and fatigue, termed the androgen deprivation syndrome. Primary care physicians providing follow-up care for men with prostate cancer will be faced with managing these effects. We therefore sought to estimate the incidence of these effects and, by using a control group, ascertain whether these effects were related to androgen deprivation itself.
\end{abstract}

\begin{abstract}
Methods-We assessed the risk of physician diagnoses of depression, cognitive impairment, or constitutional symptoms in Medicare data following androgen deprivation using a sample of 50613 men with incident prostate cancer and 50476 men without cancer, from 1992 through 1997, in the linked Surveillance, Epidemiology, and End Results-Medicare database. Cox proportional hazards regression was used to adjust for confounding variables.
\end{abstract}

Results-Of men surviving at least 5 years after diagnosis, $31.3 \%$ of those receiving androgen deprivation developed at least 1 depressive, cognitive, or constitutional diagnosis compared with $23.7 \%$ in those who did not $(P<.001)$. After adjustment for variables such as comorbidity, tumor characteristics, and age, the risks associated with androgen deprivation were substantially reduced or abolished: relative risk (RR) for depression diagnosis, 1.08 (95\% confidence interval [CI], 1.02-1.15); RR for cognitive impairment, 0.99 (95\% CI, 0.94-1.04); and RR for constitutional symptoms, 1.17 (95\% CI, 1.13-1.22).

Conclusion-Depressive, cognitive, and constitutional disorders occur more commonly in patients receiving androgen deprivation, but this appears to be primarily because patients receiving androgen deprivation are older and have more comorbid conditions and more advanced cancers.

Androgen deprivation has become a common treatment for prostate cancer, with nearly half of all men with the disease receiving the therapy at some point in their course of treatment. ${ }^{1}$, 2 Androgen deprivation is increasingly used for early stages of prostate cancer, exposing men to the therapy for longer periods. ${ }^{2,3}$ In light of these trends, concerns about the toxic effects of androgen deprivation have been raised. ${ }^{4,5}$ Sexual dysfunction is a well-documented adverse effect of androgen deprivation. ${ }^{6-8}$ However, there is increasing recognition of a spectrum of other less specific adverse effects such as depression, anxiety, malaise, fatigue, and memory

Correspondence: Vahakn B. Shahinian, MD, MS, University of Texas Medical Branch, Department of Internal Medicine, John Sealy Annex 4.200, 301 University Blvd, Galveston, TX 77555-0562 (vbshahin@utmb.edu).

The interpretation and reporting of these data are the sole responsibility of the authors.

Financial Disclosure: None.

Role of the Sponsor: The funding bodies had no role in data extraction and analyses, in the writing of the manuscript, or in the decision to submit the manuscript for publication. 
difficulties, which some authors have termed the androgen deprivation syndrome. ${ }^{9-16}$ The burden of managing these adverse effects will likely be faced by primary care physicians, who provide much of the long-term follow-up care for patients with prostate cancer. ${ }^{17,18}$ It is therefore important to provide an estimate of the prevalence of these adverse effects. Previous studies examining these issues have been small, single center, and without controls. ${ }^{19}$ Because many of these effects could plausibly occur secondary to the cancer, controls are necessary to ascertain the impact of the androgen deprivation itself. We examined these adverse effects in a large, population-based sample using the linked Surveillance, Epidemiology and End-Results (SEER)-Medicare database.

\section{Methods}

\section{Data Sources}

Data used for this study derived from the linked SEER-Medicare database. ${ }^{20,21}$ The SEER program consists of a group of population-based tumor registries in selected geographic areas, from 11 states, covering approximately $14 \%$ of the US population. Medicare is a federal program that covers health services for $97 \%$ of persons 65 years and older. It provides data in the form of claims submitted by providers for reimbursement, which include information on diagnoses and the service, testing, or procedure carried out.

\section{Study Subjects}

Prostate Cancer Cases-The study protocol was approved by the local institutional review board. All men 66 years and older first diagnosed as having prostate cancer in the years 1992 through 1997 were selected, for a total of 92474 subjects. To ensure complete information, patients not enrolled in both Medicare Part A and Part B for the 12 months before and after their cancer diagnosis (13 352 cases), members of a health maintenance organization (17 275 cases), or those whose cancer was diagnosed by autopsy or on a death certificate (1076 cases) were excluded. Subjects with prostate cancer were divided into 2 groups: those who underwent androgen deprivation (defined as receipt of at least 1 dose of a gonadotropin-releasing hormone $[\mathrm{GnRH}]$ agonist or orchiectomy within 6 months of diagnosis) and those who did not receive androgen deprivation (defined as not receiving GnRH agonists or undergoing orchiectomy at any time following the diagnosis). This excluded 10158 patients who started therapy with GnRH agonists or underwent orchiectomy 6 months or more after diagnosis. Overall, 50613 patients with prostate cancer were available for the primary sample, with follow-up through 2001.

Noncancer Controls-As another comparison group, we developed a noncancer cohort from the SEER-Medicare data, which also includes a file containing a 5\% sample of Medicare beneficiaries residing in the SEER areas who do not have any cancer diagnosis. We selected men 66 years and older who were resident in a SEER area during the study period, had continuous Part A and Part B Medicare coverage, and were not enrolled in a health maintenance organization for at least 3 consecutive years between 1991 and 1997. The initial study entry year for these men was assigned randomly to match the distribution for the year of diagnosis of cancer in men in the prostate cancer cohort. In this way, we constructed a cohort of 50476 men without cancer, with follow-up through 2001.

\section{Definitions}

Details for identification of variables of interest for this study such as patient demographic and socioeconomic characteristics, comorbid conditions, cancer treatments administered, and cancer characteristics have been previously published. ${ }^{8}$ Briefly, patient demographics such as age and race and tumor characteristics such as grade and American Joint Committee on Cancer (AJCC) stage were derived from the SEER Patient Entitlement and Diagnosis Summary file. 
Socioeconomic characteristics were based on ZIP code of residence through a linkage with the 1990 US census. Comorbidity was assessed using the modification of the Charlson comorbidity index ${ }^{22}$ by Klabunde et al, ${ }^{23}$ based on information from Medicare inpatient and outpatient claims. ${ }^{22}$ Cancer treatments were also based on information from Medicare claims. Diagnoses that were potentially compatible with the androgen deprivation syndrome were grouped into 3 categories: depressive, cognitive, or constitutional disorders (Table 1). A disorder was deemed to occur if it was listed as a diagnosis at least once in a Medicare inpatient, outpatient, or physician claim.

\section{Statistical Analysis}

The $\chi^{2}$ test was used to compare the proportions of patients with a diagnosis of a depressive, cognitive, or constitutional disorder in the 12 months prior to or during the 6- to 60-month period following the diagnosis of prostate cancer or study entry among patients with prostate cancer who received androgen deprivation, patients with prostate cancer who did not receive androgen deprivation, and patients without prostate cancer. To ensure complete follow-up for this analysis, patients who died or lost Medicare Part A or B coverage during the 60 months following diagnosis or study entry were excluded. In addition, patients who were diagnosed as having a disorder in the first 6 months of diagnosis of cancer or study entry were excluded because it was believed that these outcomes were less likely to be related to the androgen deprivation therapy.

Survival analyses were performed using Cox proportional hazards regression for patients with prostate cancer, with the dependent variable being time to first diagnosis of a disorder. Data were censored at death, end of follow-up period, or switch away from Medicare coverage. Patients who died or were diagnosed as having a disorder in the 6 months following diagnosis of cancer were excluded (inclusion of patients who developed disorders in the first 6 months following diagnosis did not substantially alter our results). The following characteristics were entered as independent variables in the model: use of androgen deprivation, age at diagnosis or study entry, race, education, income, SEER region, comorbidity, diagnosis of a depressive, cognitive or constitutional disorder in the 12 months prior to diagnosis or study entry, number of provider visits in the 12 months prior to diagnosis or study entry, cancer grade, cancer stage, use of radiation therapy, and use of radical prostatectomy. All analyses were performed using SAS version 8.2 (SAS Institute Inc, Cary, NC). All tests of statistical significance were 2 sided, with $P<.05$ considered to be statistically significant.

\section{Results}

\section{Patient and Cancer Characteristics}

Table 2 presents characteristics of the 50613 men constituting the prostate cancer cohort, categorized by whether they received androgen deprivation (31\% of the patients received androgen deprivation). Patients with prostate cancer who received androgen deprivation were older, with a median age of 75 years (vs 72 years), and $27.6 \%$ of patients were 80 years and older (vs 13.7\%). Patients receiving androgen deprivation also tended to have more advanced and aggressive cancers, with 20.0\% having AJCC stage IV tumors (vs 2.9\%) and 33.9\% having poorly differentiated tumors (vs $13.3 \%$ ).

The characteristics of the 50476 noncancer controls are also listed in Table 2. Their median age was 72 years. They tended to have slightly more comorbid conditions, with $7.5 \%$ of patients with a comorbidity index of 3 or higher vs $6.7 \%$ and $5.6 \%$ in the prostate cancer with androgen deprivation and without androgen deprivation groups, respectively. 


\section{Proportion of Patients with Depressive, Cognitive, and Constitutional Disorders}

Depressive disorders during the 6- to 60-month period following diagnosis of cancer or study entry developed in a similar proportion of patients without cancer $(9.6 \%$; $95 \%$ confidence interval [CI], 9.2\%-9.9\%) and patients with prostate cancer who did not receive androgen deprivation $(9.5 \%$; $95 \% \mathrm{CI}, 9.1 \%-9.9 \%)$ but were significantly more common in patients with prostate cancer receiving androgen deprivation $(12.1 \%$; 95\% CI, 11.3\%-12.8\%) (Table 3). Cognitive disorders during the 6- to 60-month period following diagnosis or study entry were most common in the prostate cancer group who received androgen deprivation (13.9\%; $95 \%$ CI, $13.1 \%-14.7 \%)$, intermediate in the prostate cancer group who did not receive androgen deprivation $(10.2 \%$; $95 \% \mathrm{CI}, 9.8 \%-10.6 \%)$, and least common in the noncancer group (7.9\%; $95 \%$ CI, 7.6\%-8.2\%). Constitutional disorders were most common in the prostate cancer group who received androgen deprivation $(16.7 \%$; $95 \% \mathrm{CI}, 15.9 \%-17.6 \%)$, intermediate in the noncancer group (12.9\%; 95\% CI, $12.5 \%-13.3 \%)$, and least common in the prostate cancer group who did not receive androgen deprivation (11.4\%; 95\% CI, 10.9\%-11.8\%). The proportion of patients developing at least 1 depressive, cognitive, or constitutional disorder was $31.3 \%$ (95\% CI, 30.2\%-32.4\%) in the prostate cancer group who received androgen deprivation, $23.7 \%$ (95\% CI, 23.1\%-24.3\%) in the prostate cancer group who did not, and $22.9 \%$ (95\% CI, $22.4 \%-23.4 \%$ ) in the noncancer group.

\section{Risk of Depressive, Cognitive, and Constitutional Disorders Associated with Androgen Deprivation Therapy}

A Cox model was performed to assess the risks of depressive, cognitive, and constitutional disorders associated with androgen deprivation therapy. The analysis was limited to patients with prostate cancer, and the aim was to compare the risk of disorders between patients who received androgen deprivation with those who did not. Subjects were followed for a mean of 52 months following diagnosis of cancer.

Unadjusted analyses demonstrated significant increases in the risk of depressive (relative risk [RR], 1.37; 95\% CI, 1.30-1.44), cognitive (RR, 1.44; 95\% CI, 1.38-1.50), and constitutional (RR, 1.57; 95\% CI, 1.51-1.62) disorders associated with receipt of androgen deprivation. In adjusted analyses, these risks were substantially reduced or eliminated. The RR was 1.08 (95\% CI, 1.02-1.15) for depressive disorders, 0.99 (95\% CI, 0.94-1.04) for cognitive disorders, and 1.17 (95\% CI, 1.13-1.22) for constitutional disorders.

We performed a series of additional analyses. First, to examine whether the effect of androgen deprivation differed in healthier patients with earlier-stage cancers, we restricted the analyses to patients with early stage disease (AJCC stage I or II and low- or moderate-grade histologic features), age younger than 80 years at diagnosis, and a comorbidity index of 0 . The pattern was similar to the results for the analyses that included all patients. For depressive disorders, the unadjusted risk associated with androgen deprivation was 1.28 (95\% CI, 1.12-1.47), and this fell to 1.19 (95\% CI, 1.03-1.37) in the adjusted analysis. For cognitive disorders, the unadjusted risk associated with androgen deprivation was 1.29 (95\% CI, 1.13-1.46), and this fell to 1.10 (95\% CI, 0.96-1.26) in the adjusted analysis. For constitutional disorders, the unadjusted risk associated with androgen deprivation was 1.62 (95\% CI, 1.49-1.77), and this fell to 1.22 (95\% CI, 1.11-1.34) in the adjusted analysis.

Second, to examine whether the effects differed in patients receiving prolonged androgen deprivation, we analyzed the risks for depressive, cognitive, and constitutional disorders associated with use of at least 9 doses of a GnRH agonist in the first year and the risks associated with orchiectomy performed in the first 6 months of diagnosis. For orchiectomy, there was a slightly higher risk for depressive disorders (RR, 1.15; 95\% CI, 1.03-1.27). Otherwise, the results were essentially unchanged from the analyses for overall androgen deprivation. 
Third, we performed another Cox model including both patients with and without prostate cancer. This analysis included adjustment for the same variables included in the previous Cox models except for the cancer-related variables. Patients without cancer were the reference group. For patients with prostate cancer receiving androgen deprivation compared with patients without cancer, the RR was 1.13 (95\% CI, 1.08-1.19) for depressive disorders and 1.26 (95\% CI, 1.21-1.30) for constitutional disorders. There were no significant differences in the risks of depressive or constitutional disorders between patients with prostate cancer who did not receive androgen deprivation vs the noncancer controls. The risk of cognitive disorders was significantly increased in patients with prostate cancer with and without androgen deprivation compared with the noncancer group, with RRs of 1.32 (95\% CI, 1.27-1.38) and 1.20 (95\% CI, 1.15-1.24), respectively.

\section{Comment}

To our knowledge, this study is the first population-based analysis of depressive, cognitive, and constitutional diagnoses in patients with prostate cancer receiving androgen deprivation. In unadjusted analyses, the risks of depressive, cognitive, and constitutional disorders were substantially increased in patients receiving androgen deprivation. However, after adjustment for potentially confounding variables such as tumor grade and comorbidity, the risks declined substantially. There were still small but significant increases in the risks of depressive and constitutional disorders in patients with prostate cancer who received androgen deprivation compared with those who did not.

Potential complications of androgen deprivation have received increasing attention because of recent trends in the use of this therapy. Despite no demonstrated survival benefit, androgen deprivation therapy is now commonly used for early primary treatment of localized and locally advanced disease, as well as for biochemical recurrence following radical prostatectomy. ${ }^{2}$ In addition, there has been a dramatic rise in its use as adjuvant therapy combined with radiation in locally advanced or high-risk subsets of localized disease in the face of clinical trials showing improved survival with this regimen. $3,24,25$ These changes have led to more men with prostate cancer being exposed to androgen deprivation and for longer periods.

There are good theoretical reasons to suspect that androgen deprivation may cause the emotional disturbances, fatigue, and memory difficulties that have collectively been termed the androgen deprivation syndrome. Although data are conflicting, some studies of elderly men have demonstrated an association between low testosterone levels and depressive illness. 26,27 Furthermore, a study of 4 hypogonadal men with depression refractory to conventional therapy showed dramatic improvement following testosterone replacement, with relapses occurring in 3 after switching to placebo. ${ }^{28}$ Low testosterone levels are also associated with poorer cognitive function. ${ }^{29}$ A controlled trial of testosterone administration in 25 healthy older men showed improvements in spatial and verbal memory. ${ }^{30}$ Finally, fatigue could plausibly result from reductions in muscle mass that occur following androgen deprivation. 31,32

Despite biological plausibility, studies specifically addressing the association between androgen deprivation in prostate cancer and subsequent depressive, cognitive, or constitutional symptoms are limited. Previous reports examining the risk of depression in patients with prostate cancer receiving androgen deprivation are restricted to case series or small cohort studies without a control group. ${ }^{10,12,13}$ The largest study, involving 45 men receiving androgen deprivation, demonstrated a prevalence of major depression of $12.8 \%$, which the authors noted to be 8 times the national rate of depression in men. ${ }^{13}$ Results from studies examining the effect of androgen deprivation on cognitive function in patients with prostate cancer are conflicting. ${ }^{14,33}$ A clinical trial assessed cognitive function in 82 men with prostate 
cancer randomized to active treatment with androgen deprivation vs observation alone. ${ }^{14}$ Nearly $50 \%$ of the men in the treatment arm developed a decline in the results of 1 or more cognitive tests after 6 months of therapy, whereas no declines were noted in the observation group. In contrast, an observational study of 25 men receiving androgen deprivation together with radiation showed no decline in cognitive function after 12 months of therapy. ${ }^{33}$ Finally, only 1 uncontrolled study specifically examined fatigue in the setting of androgen deprivation for prostate cancer. ${ }^{15} \mathrm{~A}$ total of 62 men with prostate cancer were assessed using a fatigue questionnaire at baseline and after 3 months of therapy with a GnRH agonist, and 66\% described a worsening in their fatigue score after therapy was initiated.

Recent reviews on complications of androgen deprivation in patients with prostate cancer note that effects such as depression and fatigue can and have been attributed to the cancer itself or to other medical comorbid conditions. 7,19 In this regard, cancers of various sites have been associated with depression, fatigue, and cognitive dysfunction, with worse symptoms in patients with more advanced cancers. 34,35 Similar associations have been described with chronic medical illness in the elderly. ${ }^{36,37}$ Evidence from our study shows that although depressive, cognitive, and constitutional disorders occur more commonly in patients receiving androgen deprivation, this is primarily because patients receiving androgen deprivation are older, have more comorbid conditions, and have more advanced cancers (Table 2). ${ }^{2}$ These disorders therefore may not be causally related to the androgen deprivation itself. After adjustment, the risks of these disorders were substantially reduced or abolished. This remained true even when we limited the analyses to the risks associated with prolonged androgen deprivation in the form of orchiectomy. The small residual increases in risk of depressive or constitutional disorders in adjusted analyses may represent true but modest effects of androgen deprivation or may plausibly be due to incomplete adjustment and residual confounding.

The main limitation of this study is the issue of ascertainment of the disorders. A Medicare claims approach is known to have poor sensitivity for diagnoses not associated with a procedure. ${ }^{38}$ In addition, patients often do not report symptoms such as fatigue or depression to their physicians. ${ }^{39}$ As such, rates of the disorders noted in Table 3 probably represent underestimates of the true prevalence of these conditions. However, because the method of ascertainment of the disorders was similar among all the groups studied, the RRs generated should still be valid. In addition, the large sample size in our study allowed relatively precise estimates of the risks, making it unlikely that a large effect of androgen deprivation was missed because of insufficient power. Nevertheless, we cannot exclude the presence of subtle effects of androgen deprivation that require formal cognitive or psychological testing to detect.

There are a number of implications from this study. The presence of substantial confounding in the assessment of the effect of androgen deprivation on the occurrence of depressive, cognitive, and constitutional disorders underscores the need for controls in all future studies examining these issues. The risks of depression or constitutional effects directly attributable to androgen deprivation are at best modest and should not preclude the use of this therapy in settings in which its benefits are clear. Nevertheless, these conditions are especially common in patients with prostate cancer receiving androgen deprivation, affecting at least $30 \%$ of men over a 5-year period. Primary care physicians and urologists treating these patients should therefore be aware of the potential for these disorders to develop and encourage patients to report relevant symptoms. Effective treatments are available for some of these conditions and may help improve the quality of life for patients with prostate cancer.

\section{Acknowledgements}

Funding/Support: This work was supported in part by grants P50CA105631 and R24HS011618 from the Public Health Service, Bethesda, Md. 
This study used the linked SEER-Medicare database. The authors acknowledge the efforts of the Applied Research Program, National Cancer Institute, Rockville, Md; the Office of Research, Development and Information, Centers for Medicare \& Medicaid Services, Washington, DC; Information Management Services Inc, Silver Spring, Md; and the SEER Program tumor registries in the creation of the SEER-Medicare database.

\section{References}

1. Meng MV, Grossfeld GD, Sadetsky N, Mehta SS, Lubeck DP, Carroll PR. Contemporary patterns of androgen deprivation therapy use for newly diagnosed prostate cancer. Urology 2002;60:7-12. [PubMed: 12231037]

2. Shahinian VB, Kuo YF, Freeman JL, Orihuela E, Goodwin JS. Increasing use of gonadotropinreleasing hormone agonists for localized prostate cancer. Cancer 2005;103:1615-1624. [PubMed: 15742331]

3. Cooperberg MR, Grossfeld GD, Lubeck DP, Carroll PR. National practice patterns and time trends in androgen ablation for localized prostate cancer. J Natl Cancer Inst 2003;95:981-989. [PubMed: 12837834]

4. Soloway MS. Timing of androgen deprivation for prostate cancer: benefits versus side effects-a patient-physician dialogue. Urology 2002;60:735-737. [PubMed: 12429286]

5. Milbank AJ, Dreicer R, Klein EA. Hormonal therapy for prostate cancer: primum non nocere. Urology 2002;60:738-741. [PubMed: 12429287]

6. Schroder FH. Endocrine treatment of prostate cancer-recent developments and the future, 1: maximal androgen blockade, early vs delayed endocrine treatment and side-effects. BJU Int 1999;83:161-170. [PubMed: 10233478]

7. Higano CS. Side effects of androgen deprivation therapy: monitoring and minimizing toxicity. Urology 2003;61:32-38. [PubMed: 12667885]

8. Shahinian VB, Kuo YF, Freeman JL, Goodwin JS. Risk of fracture after androgen deprivation for prostate cancer. N Engl J Med 2005;352:154-164. [PubMed: 15647578]

9. Holzbeierlein JM, McLaughlin MD, Thrasher JB. Complications of androgen deprivation therapy for prostate cancer. Curr Opin Urol 2004;14:177-183. [PubMed: 15069309]

10. Rosenblatt DE, Mellow A. Depression during hormonal treatment of prostate cancer. J Am Board Fam Pract 1995;8:317-320. [PubMed: 7572297]

11. Freeman MP, Freeman SA. Treatment of leuprolide-induced depression with intramuscular testosterone: a case report. J Clin Psychiatry 2003;64:341-343. [PubMed: 12716284]

12. Higano CS, Ellis W, Russell K, Lange PH. Intermittent androgen suppression with leuprolide and flutamide for prostate cancer: a pilot study. Urology 1996;48:800-804. [PubMed: 8911533]

13. Pirl WF, Siegel GI, Goode MJ, Smith MR. Depression in men receiving androgen deprivation therapy for prostate cancer: a pilot study. Psychooncology 2002;11:518-523. [PubMed: 12476433]

14. Green HJ, Pakenham KI, Headley J, et al. Altered cognitive function in men treated for prostate cancer with luteinizing-hormone-releasing hormone analogues and cyproterone acetate: a randomized controlled trial. BJU Int 2002;90:427-432. [PubMed: 12175403]

15. Stone P, Richards M, Hardy J. Fatigue in patients with cancer. Eur J Cancer 1998;34:1670-1676. [PubMed: 9893650]

16. McDermed J, Strum S, Scholz M. The androgen deprivation syndrome (ADS): the incidence and severity in prostate cancer (PC) patients (PTS) receiving hormone blockade (HB) [abstract]. Proc Am Soc Clin Oncol 1998; 17:315a.

17. Freedman A, Hahn G, Love N. Follow-up after therapy for prostate cancer. Postgrad Med 1996;100:125-129. 133-34, 136. [PubMed: 8795650]

18. Sunga AY, Eberl MM, Oeffinger KC, Hudson MM, Mahoney MC. Care of cancer survivors. Am Fam Physician 2005;71:699-706. 713-714. [PubMed: 15742907]

19. Thompson CA, Shanafelt TD, Loprinzi CL. Andropause: symptom management for prostate cancer patients treated with hormonal ablation. Oncologist 2003;8:474-487. [PubMed: 14530501]

20. Warren JL, Klabunde CN, Schrag D, Bach PB, Riley GF. Overview of the SEER-Medicare data: content, research applications, and generalizability to the United States elderly population. Med Care 2002;40(8):IV-3-18. 
21. Potosky AL, Riley GF, Lubitz JD, Mentnech RM, Kessler LG. Potential for cancer related health services research using a linked Medicare-tumor registry database. Med Care 1993;31:732-748. [PubMed: 8336512]

22. Charlson ME, Pompei P, Ales KL, MacKenzie CR. A new method of classifying prognostic comorbidity in longitudinal studies: development and validation. J Chronic Dis 1987;40:373-383. [PubMed: 3558716]

23. Klabunde CN, Potosky AL, Legler JM, Warren JL. Development of a comorbidity index using physician claims data. J Clin Epidemiol 2000;53:1258-1267. [PubMed: 11146273]

24. D'Amico AV, Manola J, Loffredo M, Renshaw AA, DellaCroce A, Kantoff PW. 6-Month androgen suppression plus radiation therapy vs radiation therapy alone for patients with clinically localized prostate cancer: a randomized controlled trial. JAMA 2004;292:821-827. [PubMed: 15315996]

25. Lawton CA, Winter K, Murray K, et al. Updated results of the phase III Radiation Therapy Oncology Group (RTOG) trial 85-31 evaluating the potential benefit of androgen suppression following standard radiation therapy for unfavorable prognosis carcinoma of the prostate. Int J Radiat Oncol Biol Phys 2001;49:937-946. [PubMed: 11240234]

26. Seidman SN, Walsh BT. Testosterone and depression in aging men. Am J Geriatr Psychiatry 1999;7:18-33. [PubMed: 9919317]

27. Shores MM, Sloan KL, Matsumoto AM, Moceri VM, Felker B, Kivlahan DR. Increased incidence of diagnosed depressive illness in hypogonadal older men. Arch Gen Psychiatry 2004;61:162-167. [PubMed: 14757592]

28. Seidman SN, Rabkin JG. Testosterone replacement therapy for hypogonadal men with SSRIrefractory depression. J Affect Disord 1998;48:157-161. [PubMed: 9543205]

29. Yaffe K, Lui LY, Zmuda J, Cauley J. Sex hormones and cognitive function in older men. J Am Geriatr Soc 2002;50:707-712. [PubMed: 11982672]

30. Cherrier MM, Asthana S, Plymate S, et al. Testosterone supplementation improves spatial and verbal memory in healthy older men. Neurology 2001;57:80-88. [PubMed: 11445632]

31. Smith MR, Finkelstein JS, McGovern FJ, et al. Changes in body composition during androgen deprivation therapy for prostate cancer. J Clin Endocrinol Metab 2002;87:599-603. [PubMed: 11836291]

32. Smith JC, Bennett S, Evans LM, et al. The effects of induced hypogonadism on arterial stiffness, body composition, and metabolic parameters in males with prostate cancer. J Clin Endocrinol Metab 2001;86:4261-4267. [PubMed: 11549659]

33. Salminen E, Portin R, Korpela J, et al. Androgen deprivation and cognition in prostate cancer. Br J Cancer 2003;89:971-976. [PubMed: 12966411]

34. Rao A, Cohen HJ. Symptom management in the elderly cancer patient: fatigue, pain, and depression. J Natl Cancer Inst Monogr 2004;32:150-157. [PubMed: 15263059]

35. Valentine AD, Meyers CA. Cognitive and mood disturbance as causes and symptoms of fatigue in cancer patients. Cancer 2001;92:1694-1698. [PubMed: 11598889]

36. Leon FG, Ashton AK, D'Mello DA, et al. Depression and comorbid medical illness: therapeutic and diagnostic challenges. J Fam Pract 2003 Dec;(suppl):S19-S33. [PubMed: 14693075]

37. Raj A. Depression in the elderly: tailoring medical therapy to their special needs. Postgrad Med 2004;115:26-28. 37-42. [PubMed: 15216572]

38. Potosky AL, Warren JL, Riedel ER, Klabunde CN, Earler CC, Begg CN. Measuring complications of cancer treatment using the SEER-Medicare data. Med Care 2002;40:IV-62-68.

39. Davidson JRT, Melzer-Brady SE. The under recognition and undertreatment of depression: what is the breadth and depth of the problem? J Clin Psychiatry 1999;60:4-9. [PubMed: 10326869] 


\section{Definition of Disorders}

Disorder *

Depressive

Major depression

Sleep disturbance related to depression

Depressive psychoses

Depression with anxiety

Adjustment disorder with depression

Miscellaneous depressive disorders

Cognitive

Senile dementia

Organic or drug-related memory disturbances

Cerebral degenerations (eg, Alzheimer disease)

Constitutional

Fatigue/malaise

Anorexia/cachexia/weight loss

Abnormal weight gain

Debility

Abbreviation: ICD-9, International Classification of Diseases, Ninth Revision.

A disorder was deemed to occur if it was listed as a diagnosis in at least 1 claim in any of the outpatient, inpatient, or provider Medicare claims files.

\section{Definition}

ICD-9 diagnosis codes 296.2 and 296.3

ICD-9 diagnosis codes $307.41,307.42,307.43,307.44$

ICD-9 diagnosis codes 296.9 and 298.0

ICD-9 diagnosis codes 300.4

ICD-9 diagnosis codes 309.0, 309.1, 309.28

ICD-9 diagnosis codes 290.13, 290.21, 292.84, 296.5, 296.6, 296.7, 301.10, 301.12, 301.13,

311

ICD-9 diagnosis codes 290.xx except 290.13 and 290.21

ICD-9 diagnosis codes 292.82, 292.83, 294, 310.1

ICD-9 diagnosis codes 331.xx

ICD-9 diagnosis code 780.79

ICD-9 diagnosis codes 783.0, 783.1, 783.21, 783.22, 799.4

ICD-9 diagnosis code 783.1

ICD-9 diagnosis codes 300.5, 797, 799 


\section{Patient and Cancer Characteristics ${ }^{*}$}

Table 2

Prostate Cancer Cases: Androgen Deprivation Therapy

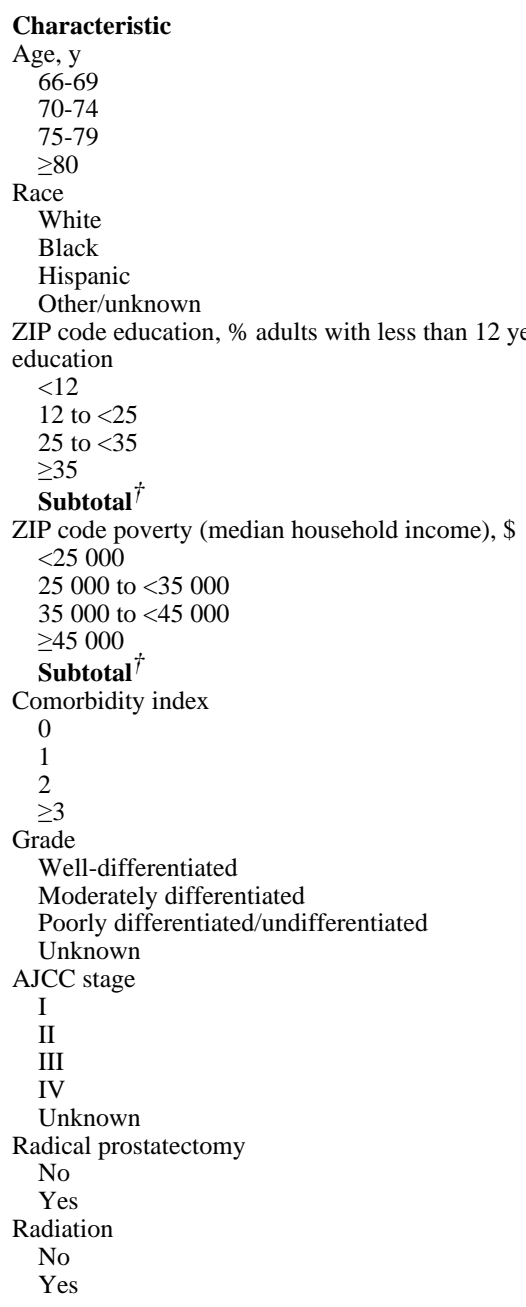

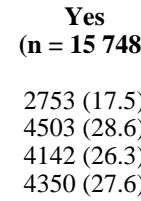

$13100(83.2)$

$1485(9.4)$

$257(1.6)$

$906(5.8)$

\section{$3419(23.0)$ \\ $6796(45.8)$ \\ $2709(18.3)$ \\ $1910(12.9)$}

14834

3181 (21.5)

$4454(30.1)$

$3799(25.7)$

$3348(22.6)$

14782

$11551(73.3)$

$2371(15.1)$

$772(4.9)$

1054 (6.7)

$1185(7.5)$

$7738(49.1)$

$5343(33.9)$

$1482(9.4)$

$96(0.6)$

$4779(30.3)$

$1548(9.8)$

$3152(20.0)$

$6173(39.2)$

$14102(89.5)$

$1646(10.5)$

$11297(71.7)$

4451 (28.3)
Noncancer Controls ( $n=50$ 476)

$17621(34.9)$

$14191(28.1)$

9459 (18.7)

$9205(18.2)$

42249 (83.7)

3017 (6.0)

$1228(2.4)$

$3982(7.9)$

$9362(23.4)$

$18500(46.2)$

$7226(18.0)$

$4975(12.4)$

40063

$8512(21.3)$

$12329(30.9)$

10354 (25.9)

$8766(21.9)$

39961

$37575(74.4)$

$6770(13.4)$

$2322(4.6)$

3809 (7.5)

Abbreviation: AJCC, American Joint Committee on Cancer.

$4775(13.7)$

$1288(3.7)$

1936 (5.6)

6506 (18.7)

$20910(60.0)$

4651 (13.3)

2798 (8.0)

1397 (4.0)

$12672(36.3)$

4922 (14.1)

1002 (2.9)

14872 (42.7)

24782 (71.1)

10083 (28.9)

$21000(60.2)$

13865 (39.8)

* Data are given as number (percentage) of patients unless otherwise specified.

${ }^{\dagger}$ Missing data. 


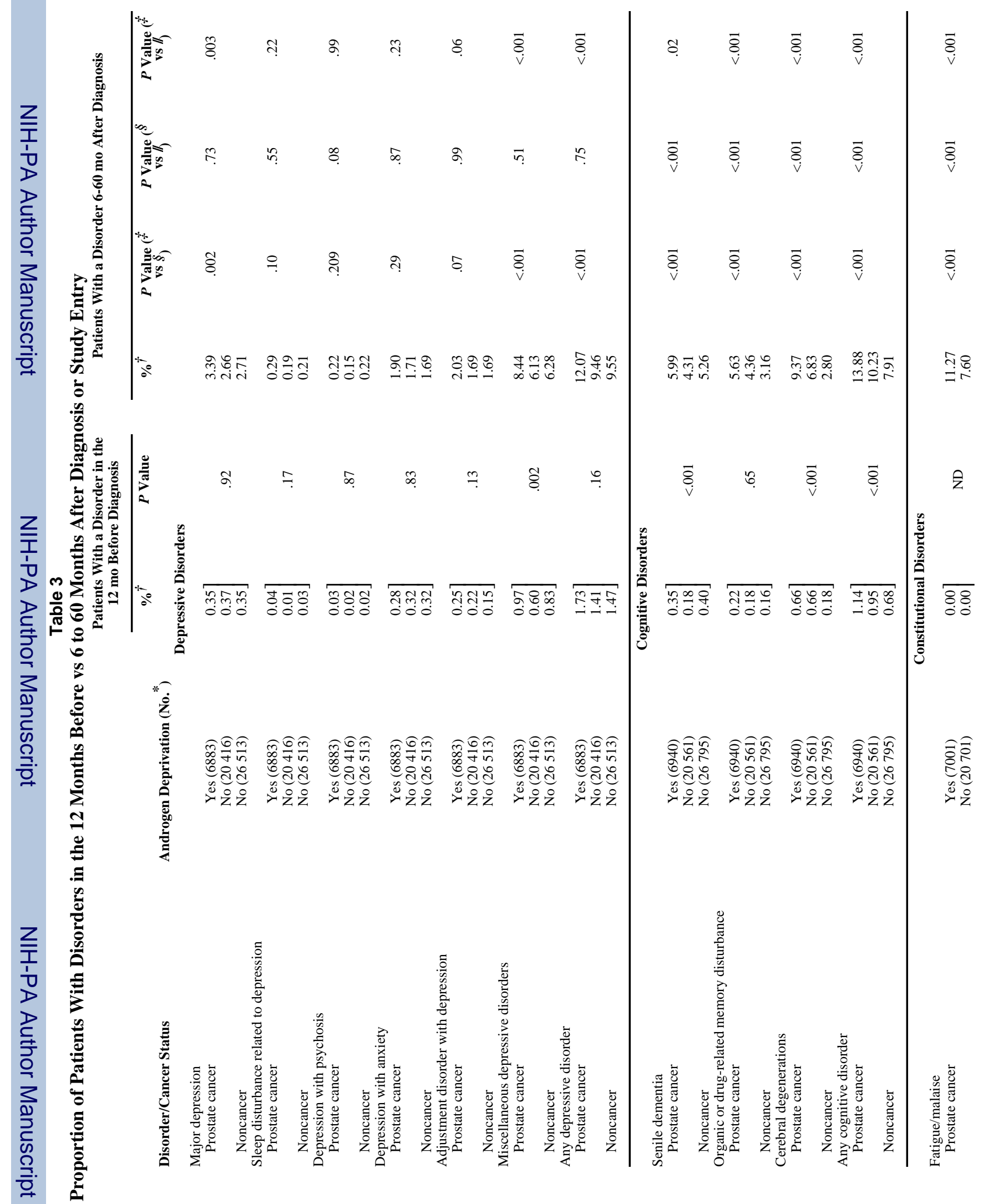




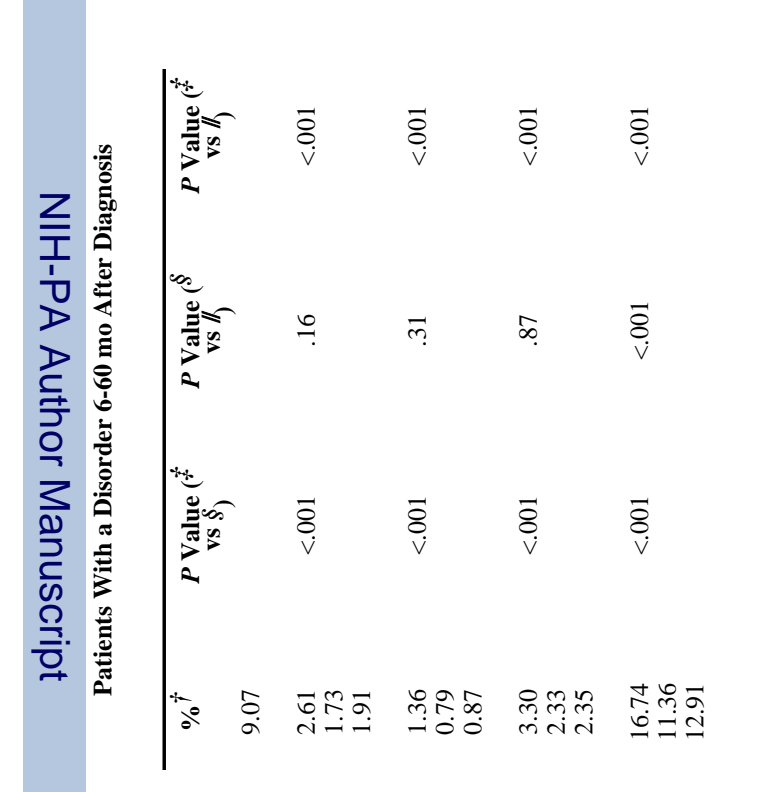

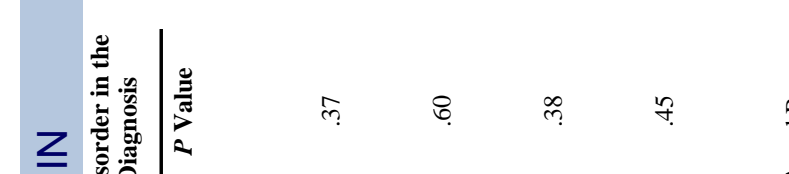

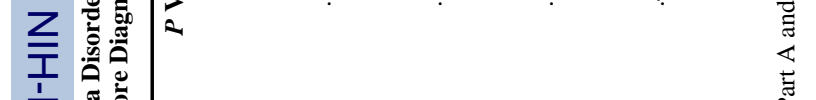

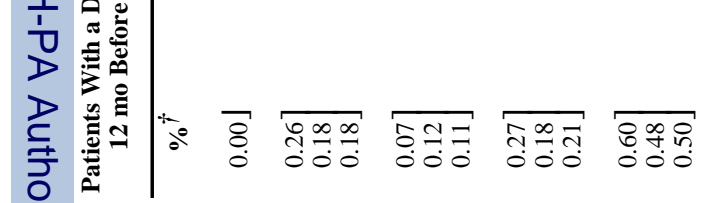

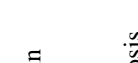

